\title{
Early Recognition of Stroke by Confrontation Visual Field Test - Is It Possible?
}

Jeniffer Jesus $^{1 *}$, Rafael Geraldes ${ }^{1}$, Manuela Amorim¹, Raquel Soares ${ }^{1}$, João Chibante-Pedro ${ }^{1}$

\section{"Correspondence author \\ Jeniffer Jesus}

Centro Hospitalar Entre o Douro e Vouga

Santa Maria da Feira

Portugal

Centro Host
Portugal

Submitted : 4 April 2020 ; Published : 24 April 2020

\begin{abstract}
Introduction: Visualfieldlossisoften thefirstsignoflesionof theanteriororposteriorvisualpathways. Screeningforvisualfield defectsshouldbeapartofallroutineeyeexaminations, mainlywhenneurologicallesionsaresuspected.Aconfrontationvisualfield (CVF) testprovidearapidandpracticalmethodofvisualfieldassessment, allowingtherecognitionofmajorneurologicaldeficits.

Materials and Research Methods: Toreportacaseofposteriorcerebralartery (PCA) strokeearlysuspectedbyCVF test.

Case-Report: A 77 year-old male presented to our emergency department with vision blurring complaints and a strange sensation of a vision loss on one side on the left eye (OS), for the previous 15 days. He explained that symptoms initiated after an episode of dizziness followed by lipothymia. He denied diplopia or headaches. Past history revealed hypertension and diabetes as cardiovascular risk factors. The best corrected visual acuity was 20/25 on right eye (OD) and 20/60 on OS and pupillary reflexes were normal. CVF test was performed and showed a decrease on left hemicampus of OS. The biomicroscopy was normal and the fundus examination showed optic nerve edema bilaterally, also documented by Optical Coherence Tomography. Considering the findings and the history, neuroimaging and a neurological observation were requested. Computerized axial tomography revealed a hypodense lesion in right occipital posterior region, with hematic density. He was hospitalized for proper treatment with the diagnosis of posterior cerebral artery ischemic stroke, with further hemorrhagic transformation.

Discussion: In acutestroke, visualdisordersmayformallorpartoftheinitialpresentation. Homonymousvisualfielddeficits are well-known almost universal characteristics of PCA ischemia, however, due to the absent of cognitive impairment or speech problems associated to many cases, the diagnosis can be challenging at early stages. Our case-report highlights the importance of using CVF test to an immediate inspection of the peripheral visual field, in order to detect acute major defects, including homonymous deficits, to understand if a further prompt neurological examination is mandatory.
\end{abstract}

Keywords: Confrontation Visual Field Test, Posterior Cerebral Artery Stroke, Homonymous Visual Field Defect, Homonymous Hemianopia

Introduction

A stroke, also referred to as a cerebral vascular accident (CVA), occurs when a clot blocks the blood supply to the brain (ischemic stroke) or when weakened blood vessel ruptures and bleeds into the surrounding brain, causing internal bleeding (hemorrhagic stroke)[1].In recent years, stroke incidence has been found to occur at an increasing rate. As the leading cause of chronic disability, the impact of strokes cannot be neglected and early recognition of them first signs is crucial. Early implementation of treatment options and interventions restores blood flow to the blocked area of the brain which quickly diminishes the risk for severe disability [2]. Even though there are many differences between ischemic and hemorrhagic strokes, the signs and symptoms for both are the same [1]. The American National Institute of Neurological 
Disorders and Stroke establish the following as the warning signs and symptoms: sudden numbness or weakness of face, arm, or leg, especially on one side of the body, sudden confusion or trouble speaking or understanding speech, sudden trouble seeing in one or both eyes, sudden trouble walking, dizziness, or loss of balance or coordination, or sudden severe headache with no known cause. The characteristics of the impairment vary mostly, depending on the type of stroke, and type and size of involved vessel. Among all the signs listed above, visual manifestations are often the first sign of lesion of posterior visual pathways [1], being visual field defects the ophthalmological abnormality most commonly reported [3-5]. Particularly, regarding occipital infarcts, previous studies have emphasized that homonymous hemianopia or quadrantanopia are the most important disability reported [4-7].

At Emergency Department of Ophthalmology, screening for visual field defects must be a part of all routine eye examinations, when neurological lesions are suspected. A confrontation visual field (CVF) test provide a rapid and practical method of visual field assessment, allowing the recognition of major neurological deficits [8-10].

\section{Case Report}

A 77 year-old male presented on our emergency department with vision blurring complaints and a strange sensation of a vision loss on one side on the left eye (OS), for the previous 15 days. He explained that symptoms initiated after an episode of dizziness followed by lipothymia. He denied diplopia or headaches. Past history revealed hypertension and diabetes as cardiovascular risk factors. The best corrected visual acuity was 20/25 on right eye (OD) and 20/60 on OS. Pupillary reflexes and eye motility were normal. CVF test was performed and showed a decrease on left hemicampus of OS. The biomicroscopy was normal and the fundus examination showed optic nerve edema bilaterally, also documented by Optical Coherence Tomography (Figure 1-2).

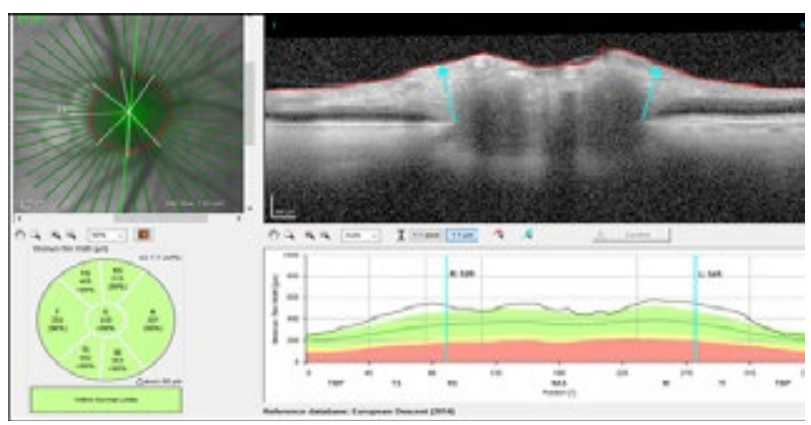

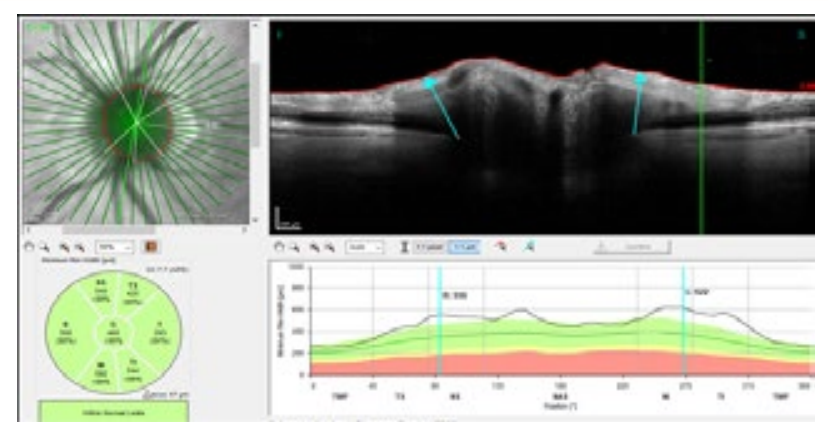

Figure 1-2: OD and OS optic nerve OCT showing bilateral papillary edema.

Considering the findings and the history, neuroimaging and a neurological opinion were requested. Computerized axial tomography revealed a hypodense lesion in right occipital posterior region, particularly in posterior cerebral artery territory and with thalamic involvement, with overlaid hematic density (Figure 3-4).
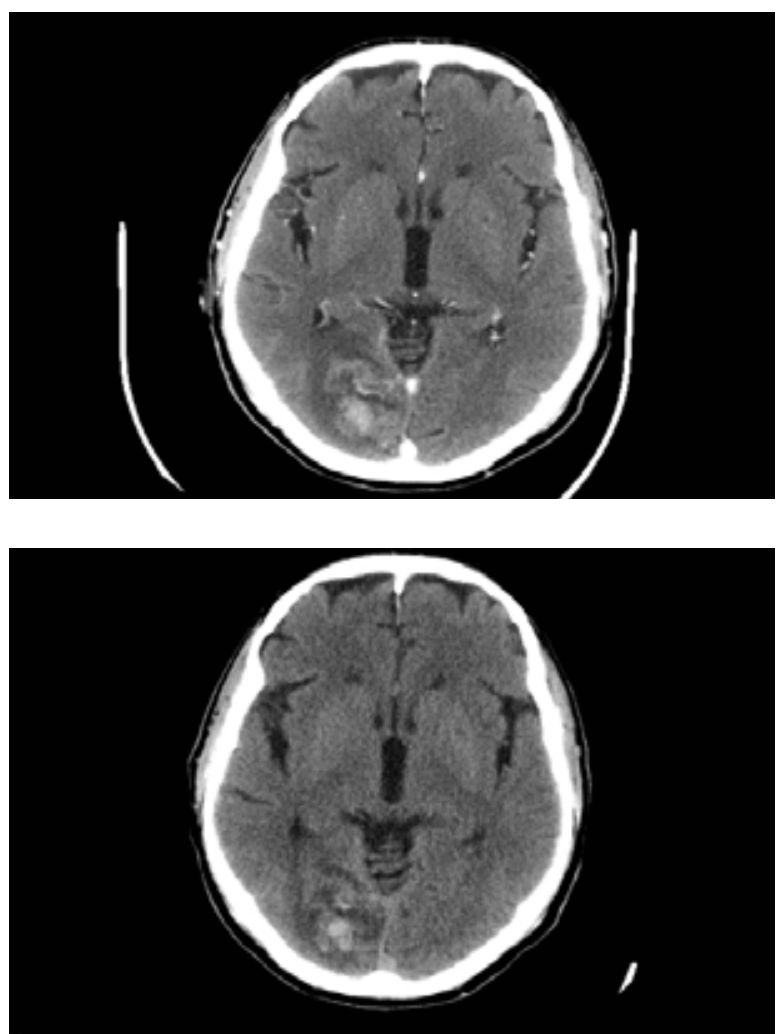

Figure 4 -5: CT showing hypodense lesion in right occipital posterior region (PCA territory), with overlaid hematic density.

To complement the study Magnetic Resonance Imaging was performed and revealed a right occipital and parasagittal cortico-subcortical lesion suggesting ischemic vascular lesion with posterior hemorrhagic transformation (Figure 5-8). He was hospitalized for proper management and treatment with the diagnosis of PCA ischemic stroke, with further hemorrhagic transformation. 

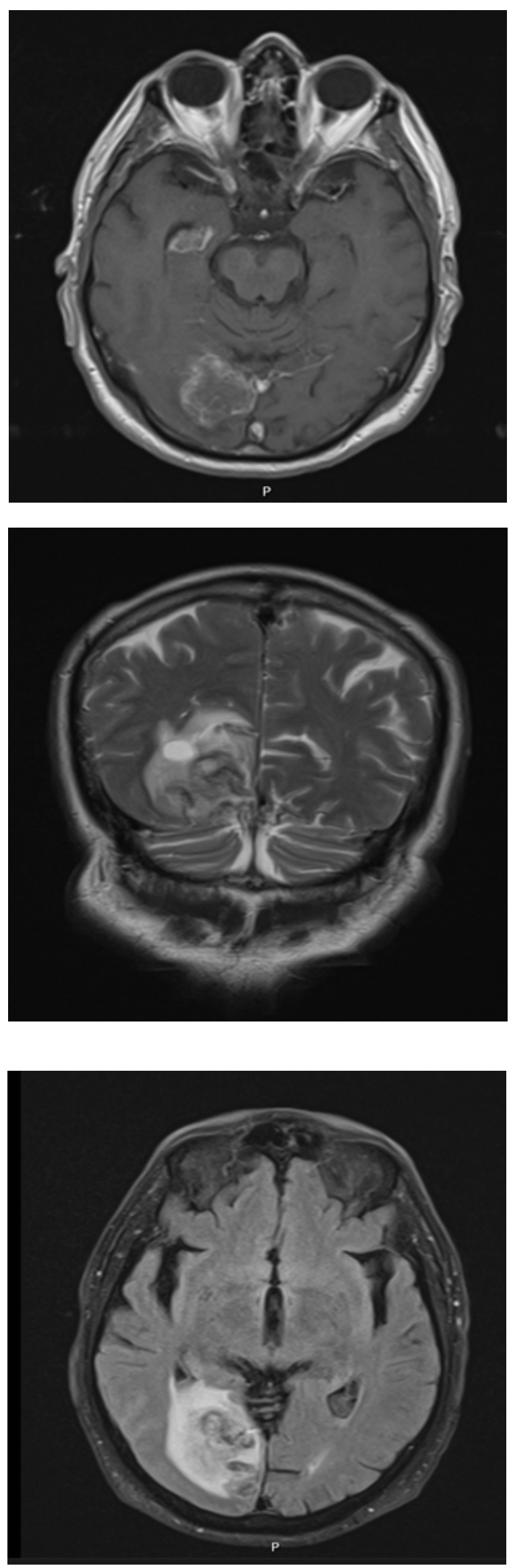

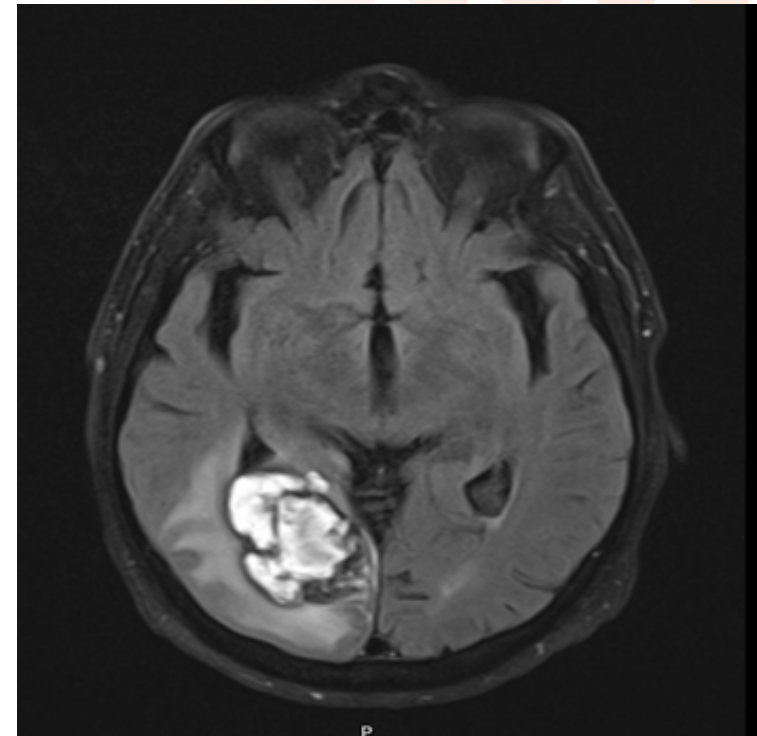

Figure 6-9: MRI reveals right occipital and parasagittal cortico-subcortical lesion suggesting ischemic vascular lesion with hemorrhagic transformation.

\section{Discussion}

In acute stroke, visual disorders may form all or part of the initial presentation. Homonymous visual field deficits are wellknown almost universal characteristics of posterior cerebral artery ischemia ${ }^{3,4}$ however, due to the absent of cognitive impairment or speech problems associated to many cases, the diagnosis can be challenging at early stages $[3,4]$.

Our case report demonstrates the importance of a complete eye examination, which includes the assessment of visual fields, during an emergency department assessment. The early recognition of a stroke may be possible at initial stages through an ophthalmological evaluation, if we are aware of neurological signs that can give us clues regarding the diagnosis, specifically regarding the location and type of lesion. Early detection of strokes is essential, to initiate a prompt and adequate in order to reduce following disabilities and deficits.

Our case-report highlights the importance of using CVF test to an immediate inspection of the peripheral visual field, in order to detect acute major defects, including homonymous deficits, to understand if a further prompt neurological examination is mandatory.

Because visual field defects can interfere with rehabilitation and is associated with a worse functional outcome in patients with stroke, we suggest that confrontation visual field test should be systematically performed in all patients during ophthalmological evaluation, mainly if there are other associated signs, such as visual loss or optic disc swelling, as in our case, to possibly better outcomes of patients with stroke, whose homonymous hemianopia or other visual field deficits were often isolated. 


\section{References}

1. Kothari R, Hall K, Brott T, Broderick J (1997) Early stroke recognition: developing an out-of-hospital NIH Stroke Scale. Acad Emerg Med 4(10): 986-990.

2. Ovbiagele B, Nguyen-Huynh MN (2011) Stroke epidemiology: advancing our understanding of disease mechanism and therapy. Neurotherapeutics 8(3): 319.

3. Wityk RJ (2017) Posterior Circulation: Large Artery Occlusive Disease and Embolism. In: Primer on Cerebrovascular Diseases. Elsevier 2017: 392-397.

4. Brandt T, Steinke W, Thie A, Pessin MS, Caplan LR (2000) Posterior Cerebral Artery Territory Infarcts: Clinical Features, Infarct Topography, Causes and Outcome1. Cerebrovasc Dis 10(3): 170-182.

5. Biousse V, Newman NJ (2017) Stroke and Eye Findings. In: Primer on Cerebrovascular Diseases. Elsevier 2017: 427-433.

6. Ramani S, Kini DV, Javali M (2018) Assessment of visual field defects in cerebrovascular disease (CVD). Indian J Clin Exp Ophthalmol 4(1): 100-106.

7. Zhang X, Kedar S, Lynn MJ, Newman NJ, Biousse V (2006) Homonymous hemianopia in stroke. J Neuroophthalmology 26(3): 180-183.

8. Kerr NM, Chew SSL, Eady EK, Gamble GD, DaneshMeyer H V (2010) Diagnostic accuracy of confrontation visual field tests. Neurology 74(15): 1184-1190.

9. Anderson AJ, Shuey NH, Wall M (2009) Rapid confrontation screening for peripheral visual field defects and extinction. Clin Exp Optom 92(1): 45-48.

10. Rowe F, Brand D, Jackson CA, et al. (2009) Visual impairment following stroke: do stroke patients require vision assessment? Age Ageing 38(2): 188-193.

Copyright: (C2020 Jeniffer Jesus. This is an open-access article distributed under the terms of the Creative Commons Attribution License, which permits unrestricted use, distribution, and reproduction in any medium, provided the original author and source are credited. 